\title{
Real-time Voltage Regulation in Distribution Systems via Decentralized PV Inverter Control
}

\author{
Weixuan Lin Robert J. Thomas Eilyan Bitar
}

\begin{abstract}
We consider the decentralized reactive power control of photovoltaic (PV) inverters spread throughout a radial distribution network. Our objective is to minimize the expected voltage regulation error, while guaranteeing the robust satisfaction of distribution system voltage magnitude and PV inverter capacity constraints. Our approach entails the offline design and the online implementation of the decentralized controller. In the offline control design, we compute the decentralized controller through the solution of a robust convex program. Under the restriction that the decentralized controller have an affine disturbance feedback form, the optimal solution of the decentralized control design problem can be computed via the solution of a finite-dimensional conic program. In the online implementation, we provide a method to implement the decentralized controller at a timescale that is fast enough to counteract the fluctuations in the system disturbance process. The resulting trajectories of $P V$ inverter reactive power injections and nodal voltage magnitudes are guaranteed to be feasible for any realization of the system disturbance under the proposed controller. We demonstrate the ability of the proposed decentralized controller to effectively regulate voltage over a fast timescale with a case study of the IEEE 123-node test feeder.
\end{abstract}

\section{INTROUDCTION}

The installation of rooftop and community solar facilities continues to increase in the United States. In California, for example, approximately $40 \%$ of all electricity demand was served by solar energy on the afternoon of May 13, 2017 [1]. Meanwhile, the increasing penetration of rooftop and community solar resources brings new challenges to voltage regulation in distribution networks [2], including overvoltage in distribution networks and deteriorated power quality due to rapid fluctuation in feeder voltage magnitudes. Traditional techniques for voltage regulation, such as the deployment of on-load tap changing (OLTC) transformers and shunt capacitors, are limited in their ability to address these challenges. Specifically, the tap positions of OLTC transformers cannot be changed at a fast time-scale due to mechanical limitations [3], and shunt capacitors cannot be switched on and off frequently due

Supported in part by NSF grant ECCS-1351621, NSF grant IIP1632124, the Department of Energy under the CERTS initiative, and the Simons Institute for the Theory of Computing.

The authors are with the School of Electrical and Computer Engineering, Cornell University, Ithaca, NY, 14853, USA. E-mails: \{w1476, rjt1, eyb5\}@cornell. edu. to the large inrush current that results during switching operations [4]. Photovoltaic (PV) inverters, on the other hand, do not suffer from these limitations. Their reactive power injections can be actively controlled at a timescale that is fast enough to counteract the fluctuation in demand and PV active power supply. Our objective in this paper is to develop a systematic approach to the design of decentralized controllers for PV inverters, in order to effectively regulate network voltage profile in real-time, while guaranteeing the robust satisfaction of network and individual inverter constraints.

Related Work: A large swath of literature treats the reactive power management of PV inverters as a centralized optimal power flow (OPF) problem, which aims to minimize a network-wide objective function (e.g., voltage regulation error) subject to network and resource constraints [5]-[10]. To set its reactive power injection, each PV inverter communicates its local measurements of demand and PV active power supply to a central computer. Using this data, the central computer solves a centralized OPF problem, and subsequently transmits the optimal solution back to each inverter for local implementation. Due to the rapid variation in the active power supply from PV resources, the reactive power injections of PV inverters need to be updated repeatedly over a fast time-scale (e.g., every minute). In the presence of a large number of PV inverters, the implementation of such reactive power control methods might be impractical, as the time required for computation and communication might exceed the time-scale at which the inverter control needs to be implemented.

This challenge in practical implementation gives rise to the need for decentralized and distributed optimization methods. In particular, there has emerged a recent stream of literature developing fully decentralized optimization methods, which enable the real-time control of PV reactive power injections using only local measurements of nodal complex power injections and voltage magnitudes [11]-[16]. Under the assumption that the underlying OPF problem being solved is time-invariant, the sequence of reactive power injection profiles computed using these decentralized methods is guaranteed to asymptotically converge to a feasible injection profile that respects both the network voltage and individual inverter constraints. There 
is, however, no guarantee on the constraint-satisfaction of these methods in finite time. Distributed optimization methods, on the other hand, rely on the explicit exchange of information between neighboring controllers in computing reactive power injections from PV inverters [17][23]. Given the satisfaction of certain requirements on the communication network specific to the optimization method being used, the sequence of reactive power injection profiles computed using these distributed optimization methods is guaranteed to converge asymptotically to a globally optimal solution of the OPF problem.

The aforementioned methods rely on a static OPF formulation of the reactive power control problem. In particular, the resulting OPF problem is parameterized by static demand and PV active power supply data. As a result, the implementation of these methods requires that demand and PV active power supply remain constant within the time interval during which the resulting optimal control is applied. Such an assumption is likely to be violated in practice, as demand and PV active power supply might vary at a time-scale that is much faster than the timescale needed for the computation and communication of the control inputs. This problem is partially addressed in [24], in which the system disturbance is treated as a random vector, and the optimal open-loop PV reactive power injections are computed to minimize the expected cost of serving demand. However, the performance of the resulting controller might be poor due to absence of feedback.

Contribution: The setting we consider entails the decentralized reactive power control of PV inverters spread throughout a radial distribution network, subject to uncertainty in demand and PV active power supply. Our approach to the decentralized control of PV inverters involves the offline design and the online implementation of the decentralized controller. In the offline control design, our objective is to minimize the expected voltage regulation error, while guaranteeing the robust satisfaction of distribution system voltage magnitude and PV inverter capacity constraints. The resulting decentralized control design problem amounts to a robust convex program. In the online implementation, we deploy the decentralized controller at each individual inverter, and implement the controller at a time-scale fast enough to counteract fluctuations in demand and PV active power supply. Our primary contributions are two-fold. First, we present a systematic approach to the computation of decentralized affine controllers via the solution of a finite-dimensional conic program. ${ }^{1}$ In particular, this approach to the compu-

\footnotetext{
${ }^{1}$ It should be noted that, subsequent to the initial submission of the present paper, Jabr in [25] has independently proposed a similar approach to the design of affine disturbance-feedback control laws for PV inverters under a different linear approximation of the $\mathrm{AC}$ power flow equations.
}

tation of decentralized controllers does not require a priori knowledge of the demand and PV active power supply realizations. Second, we provide a method to implement the decentralized affine controller over arbitrarily fast timescales (up to seconds). The proposed method is guaranteed to yield trajectories of PV inverter reactive power injections and nodal voltage magnitudes that are robustly feasible.

Organization: The remainder of this paper is organized as follows. Section II describes the distribution network, load, and PV inverter models. Section III formulates the decentralized control design problem, and presents a method to compute the optimal decentralized affine controller via the solution of a finite-dimensional conic program. Section IV describes an approach to enable realtime implementation of the decentralized affine controller. Section V demonstrates the proposed techniques with a numerical study of the IEEE 123-node test feeder. Section VI concludes the paper.

Notation: Let $\mathbf{R}$ denote the set of real numbers. For two real numbers $a \leq b$, Uni $[a, b]$ denotes the uniform distribution on $[a, b]$. We denote by $I_{n}$ the $n$-by- $n$ identity matrix, by $0_{m \times n}$ the $m$-by- $n$ zero matrix, and by $1_{m \times n}$ the $m$-by- $n$ matrix of all ones. Subscripts are omitted when the underlying matrix dimension is clear from the context. We denote the trace of a square matrix $A$ by $\operatorname{Tr}(A)$. We denote the Kronecker product operator by $\otimes$. Finally, we denote by $\mathcal{K}$ a proper cone (i.e., convex, closed, and pointed with a nonempty interior). Let $\mathcal{K}^{*}$ denote its dual cone. We write $x \succeq_{\mathcal{K}} y$ to indicate that $x-y \in \mathcal{K}$. For a matrix $A$ of appropriate dimension, $A \succeq \mathcal{K} 0$ denotes its columnwise inclusion in $\mathcal{K}$.

\section{Network And Resource Models}

\section{A. Branch Flow Model}

Consider a radial distribution network whose topology is described by a rooted tree $\mathcal{G}=(\mathcal{V}, \mathcal{E})$, where $\mathcal{V}=\{0,1, \ldots, n\}$ denotes its set of nodes, and $\mathcal{E}$ its set of (directed edges) distribution lines. In particular, node 0 is defined as the root of the network, and represents the substation that connects to the external power system. Each directed distribution line admits the natural orientation, i.e., away from the root. For each distribution line $(i, j) \in \mathcal{E}$, we denote by $r_{i j}+\mathbf{i} x_{i j}$ its impedance, whose real and imaginary parts are both assumed to be strictly positive. In addition, define $I_{i j}$ as the complex current flowing from node $i$ to $j$, and $p_{i j}+\mathbf{i} q_{i j}$ as the complex power flowing from node $i$ to $j$. For each node $i \in \mathcal{V}$, let $v_{i}$ denote its voltage magnitude, and $p_{i}+\mathbf{i} q_{i}$ the complex power injection at this node. We assume that the voltage magnitude $v_{0}$ at the substation is fixed and known.

We employ the branch flow model proposed in [26], [27] to describe the steady-state, single-phase AC power flow 
equations associated with this radial distribution network. In particular, for each node $j=1, \ldots, n$, and its unique parent $i \in \mathcal{V}$, we have

$$
\begin{aligned}
-p_{j} & =p_{i j}-r_{i j} \ell_{i j}-\sum_{k:(j, k) \in \mathcal{E}} p_{j k}, \\
-q_{j} & =q_{i j}-x_{i j} \ell_{i j}-\sum_{k:(j, k) \in \mathcal{E}} q_{j k}, \\
v_{j}^{2} & =v_{i}^{2}-2\left(r_{i j} p_{i j}+x_{i j} q_{i j}\right)+\left(r_{i j}^{2}+x_{i j}^{2}\right) \ell_{i j}, \\
\ell_{i j} & =\left(p_{i j}^{2}+q_{i j}^{2}\right) / v_{i}^{2}
\end{aligned}
$$

where $\ell_{i j}=\left|I_{i j}\right|^{2}$. We note that the branch flow model is well defined only for radial distribution networks, as we require that each node $j$ (excluding the substation) have a unique parent $i \in \mathcal{V}$.

For the remainder of the paper, we consider a linear approximation of the branch flow model (1)-(4) based on the Simplified Distflow method developed in [28]. The derivation of this approximation relies on the assumption that $\ell_{i j}=0$ for all lines $(i, j) \in \mathcal{E}$, as the power losses on distribution lines are considered to be small relative to the power flows. According to [16], [29], such an approximation tends to introduce a relative model error of $1-5 \%$ in calculating power flows for practical distribution networks. Under this assumption, Eqs. (1)-(3) can be simplified to

$$
\begin{aligned}
-p_{j} & =p_{i j}-\sum_{k:(j, k) \in \mathcal{E}} p_{j k}, \\
-q_{j} & =q_{i j}-\sum_{k:(j, k) \in \mathcal{E}} q_{j k}, \\
v_{j}^{2} & =v_{i}^{2}-2\left(r_{i j} p_{i j}+x_{i j} q_{i j}\right) .
\end{aligned}
$$

The linearized branch flow Eqs. (5)-(7) can be written more compactly as

$$
v^{2}=R p+X q+v_{0}^{2} 1
$$

Here, $v^{2}=\left(v_{1}^{2}, . ., v_{n}^{2}\right), p=\left(p_{1}, . ., p_{n}\right)$, and $q=\left(q_{1}, . ., q_{n}\right)$ denote the vectors of squared nodal voltage magnitudes, real power injections, and reactive power injections, respectively. The matrices $R, X \in \mathbf{R}^{n \times n}$ are defined according to

$$
\begin{aligned}
& R_{i j}=\underset{(h, k) \in \mathcal{P}_{i} \cap \mathcal{P}_{j}}{2} \sum_{h k}, \\
& X_{i j}=\underset{(h, k) \in \mathcal{P}_{i} \cap \mathcal{P}_{j}}{2} \sum_{h k},
\end{aligned}
$$

where $\mathcal{P}_{i} \subset \mathcal{E}$ is defined as the set of edges on the unique path from node 0 to $i$. As is shown in [16], the matrices $R$ and $X$ are guaranteed to be positive definite, since the resistance and reactance of each distribution line are both strictly positive. In particular, the positive definiteness of the matrix $X$ guarantees the uniqueness of the optimal solution to the robust convex program that we analyze in Section III.

For the remainder of this paper, we impose voltage magnitude constraints of the form

$$
\underline{v}^{2} \leq v^{2} \leq \bar{v}^{2}
$$

where the allowable range of squared voltage magnitudes is defined by the lower and upper limits $\underline{v}^{2}, \bar{v}^{2} \in \mathbf{R}^{n}$.

\section{B. Photovoltaic Inverter Model}

We consider a distribution system consisting of $n$ photovoltaic (PV) inverters whose reactive power injections can be actively controlled. For the inverter at node $i$ (excluding the substation), we denote by $\xi_{i}^{I}+\mathbf{i} q_{i}^{I}$ its complex power injection, and $\bar{s}_{i}^{I}$ its nameplate apparent power capacity. Due to the intermittency of solar irradiance, we will model the active power injection $\xi_{i}^{I}$ as a random variable, whose precise specification is presented in Section II-D. Additionally, we assume that the maximum value of the random variable $\xi_{i}^{I}$ is fixed and known, which we denote by $\bar{p}_{i}^{I}$. Clearly, it must hold that $\bar{p}_{i}^{I} \leq \bar{s}_{i}^{I}$. We require that the reactive power injections respect capacity constraints of the form:

$$
\left|q_{i}^{I}\right| \leq \sqrt{\bar{s}_{i}^{I^{2}}-\xi_{i}^{I^{2}}}, \quad i=1, \ldots, n .
$$

In the sequel, it will be convenient to work with a polyhedral inner approximation of the set of feasible complex power injections from the PV inverter. Namely, we consider the following inner approximation to constraint (10):

$$
\left|q_{i}^{I}\right| \leq \bar{s}_{i}^{I}-a_{i} \xi_{i}^{I}
$$

where the coefficient $a_{i}$ is defined according to

$$
a_{i}=\left(\bar{s}_{i}^{I}-\sqrt{\bar{s}_{i}^{I^{2}}-\bar{p}_{i}^{I}}\right) / \bar{p}_{i}^{I} .
$$

Constraint (11) approximates the set of feasible complex power injections from the PV inverter from within as a trapezoid. We provide a graphical illustration of this inner approximation in Figure 1(b). The constraints in (11) can be equivalently expressed in vector form as

$$
\left|q^{I}\right| \leq \bar{s}^{I}-A \xi^{I}
$$

Here, $\xi^{I}=\left(\xi_{1}^{I}, \ldots, \xi_{n}^{I}\right), q^{I}=\left(q_{1}^{I}, \ldots, q_{n}^{I}\right)$, and $\bar{s}^{I}=$ $\left(\bar{s}_{1}^{I}, \ldots, \bar{s}_{n}^{I}\right)$ denote the vectors of active power injections, reactive power injections, and nameplate apparent power capacities of PV inverters, respectively. Also, $\left|q^{I}\right|$ denotes the element-wise absolute value of the vector $q^{I}$. The matrix $A$ is defined as $A=\operatorname{diag}\left(a_{1}, \ldots, a_{n}\right)$. 


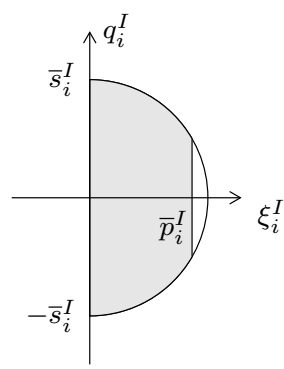

(a)

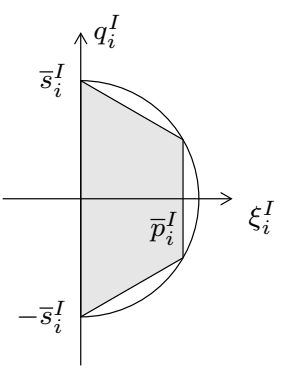

(b)
Fig. 1: The above plots depict an inverter's range of feasible complex power injections (in gray) as specified by (a) the original quadratic constraints (10) and (b) the inner linear constraints (11).

\section{Load Model}

Each node in the distribution network (apart from the substation) is assumed to have a constant power load. Accordingly, we denote by $\xi_{i}^{p} \in \mathbf{R}$ and $\xi_{i}^{q} \in \mathbf{R}$ the active and reactive power demand, respectively, at node $i$. To accommodate a priori uncertainty in realizations of active and reactive power demand, we model them as random variables - their precise specification is presented in Section II-D. The nodal active and reactive power balance equations can be expressed as

$$
\begin{aligned}
p_{i} & =\xi_{i}^{I}-\xi_{i}^{p}, \\
q_{i} & =q_{i}^{I}-\xi_{i}^{q},
\end{aligned}
$$

where $p_{i} \in \mathbf{R}$ and $q_{i} \in \mathbf{R}$ denote the net active and reactive power injections, respectively, at node $i \in\{1, \ldots, n\}$.

\section{Uncertainty Model}

As indicated earlier, we model the active power demand, reactive power demand, and PV active power supply as random variables. As a notational convention, we represent random vectors in boldface, and represent their realizations in normal face. Accordingly, we associate with each node $i$ a local disturbance defined as $\boldsymbol{\xi}_{i}=\left(\boldsymbol{\xi}_{i}^{p}, \boldsymbol{\xi}_{i}^{q}, \boldsymbol{\xi}_{i}^{\boldsymbol{I}}\right)$, which takes value in $\mathbf{R}^{3}$. We define the system disturbance according to the random vector

$$
\boldsymbol{\xi}=\left(1, \boldsymbol{\xi}_{1}, \ldots, \boldsymbol{\xi}_{n}\right),
$$

which takes value in $\mathbf{R}^{N_{\xi}}$, where $N_{\xi}=1+3 n$. Note that, in our specification of the system disturbance $\boldsymbol{\xi}$, we have included a constant scalar as its initial component. Such notational convention will prove useful in simplifying the specification of affine control policies in the sequel.

We assume that the system disturbance $\boldsymbol{\xi}$ has support $\Xi$ that is a nonempty and compact subset of $\mathbf{R}^{N_{\xi}}$, representable as

$$
\Xi=\left\{\xi \in \mathbf{R}^{N_{\xi}} \mid e_{1}^{\top} \xi=1 \text { and } W \xi \succeq_{\mathcal{K}} 0\right\},
$$

where the matrix $W \in \mathbf{R}^{\ell \times N_{\xi}}$ is known, and $e_{1}$ is the first standard basis vector in $\mathbf{R}^{N_{\xi}}$. It follows from the compactness of $\Xi$ that the second-order moment matrix

$$
M=\mathbf{E}\left[\boldsymbol{\xi} \boldsymbol{\xi}^{\top}\right]
$$

is finite-valued. We assume, without loss of generality, that $M$ is a positive definite matrix. We emphasize that our specification of the system disturbance $\boldsymbol{\xi}$ captures a large family of disturbances, including those whose support can be described as the intersection of polytopes and ellipsoids.

\section{Decentralized Control Design via Convex OPTIMIZATION}

We now describe our approach to the decentralized reactive power control of PV inverters. Specifically, we restrict our attention to the setting in which the reactive power injections from PV inverters are determined according to a decentralized affine disturbance-feedback control policy. That is, each PV inverter determines its reactive power injection according to an affine function of its local disturbance. Our objective is to minimize the expected voltage regulation error, subject to network voltage magnitude and PV inverter capacity constraints. ${ }^{2}$ The resulting decentralized control design problem amounts to a robust convex program. We show that its optimal solution can be computed by solving a finite-dimensional conic program.

\section{A. Controller Information Structure}

The controller information structure we consider in this paper is such that each PV inverter determines its reactive power injection based only on its measurements of local disturbance. ${ }^{3}$ That is, we restrict ourselves to fully decentralized disturbance-feedback controllers. The determination of an optimal decentralized controller requires the solution of an infinite-dimensional optimization problem (cf. [31]), and is, in general, computationally intractable. We thus resort to approximation, and consider decentralized controllers that are affine in the system disturbance. ${ }^{4}$ Specifically, we consider decentralized affine controllers of the form

$$
\boldsymbol{q}_{i}^{\boldsymbol{I}}=\bar{q}_{i}^{I}+Q_{i}^{I} \boldsymbol{\xi}_{i}
$$

\footnotetext{
${ }^{2}$ We note that the control design methodology proposed in this paper is general enough to accommodate power loss minimization objectives as well. We omit this treatment for the sake of brevity.

${ }^{3}$ For the ease of exposition, we assume that each PV inverter has access to perfect measurements of its local disturbance. We remark, however, that all our subsequent results can be generalized to the setting in which each PV inverter has partial linear observations of its local disturbance. We refer the readers to [30] for a detailed treatment of such problems.

${ }^{4} \mathrm{We}$ note that it is possible to improve the performance of the decentralized controller computed in this paper by enlarging the set of admissible decentralized controllers. One example is to consider decentralized controllers that are polynomial or piecewise affine in the system disturbance.
} 
for each node $i=1, \ldots, n$. Here, $\bar{q}_{i}^{I} \in \mathbf{R}$ represents the open-loop component of the local control, and $Q_{i}^{I} \in \mathbf{R}^{1 \times 3}$ is the feedback control gain matrix. We write this affine controller more compactly as

$$
\boldsymbol{q}^{\boldsymbol{I}}=Q^{I} \boldsymbol{\xi}
$$

where the matrix $Q^{I} \in \mathbf{R}^{n \times N_{\xi}}$ is given by

$$
Q^{I}=\left[\begin{array}{cccc}
\bar{q}_{1}^{I} & Q_{1}^{I} & & \\
\vdots & & \ddots & \\
\bar{q}_{n}^{I} & & & Q_{n}^{I}
\end{array}\right]
$$

We denote by $S \subseteq \mathbf{R}^{n \times N_{\xi}}$ the linear subspace of all matrices of the form specified by Eq. (14). Clearly, a matrix $Q^{I} \in \mathbf{R}^{n \times N_{\xi}}$ corresponds to a decentralized affine controller if and only if $Q^{I} \in S$.

\section{B. Design of Disturbance-feedback Affine Controllers}

Our objective is to design a decentralized affine controller that minimizes the expected voltage regulation error, while guaranteeing the satisfaction of network voltage magnitude and PV inverter capacity constraints for all possible realizations of the system disturbance $\boldsymbol{\xi}$. In what follows, we formulate the resulting decentralized control design problem as a robust convex program. In Theorem 1, we show that its optimal solution can be computed via the solution of an equivalent finite-dimensional conic program.

We first specify the affine map, which relates the system disturbance to the vectors of nodal active and reactive power injections. Namely, under the decentralized affine controller specified according to Eq. (13), we have

$$
\begin{aligned}
& \boldsymbol{p}=P \boldsymbol{\xi}, \\
& \boldsymbol{q}=Q \boldsymbol{\xi}+Q^{I} \boldsymbol{\xi},
\end{aligned}
$$

where the matrices $P$ and $Q$ are given by

$$
\begin{aligned}
& P=\left[\begin{array}{ll}
0 & I_{n} \otimes\left[\begin{array}{lll}
-1 & 0 & 1
\end{array}\right]
\end{array}\right], \\
& Q=\left[\begin{array}{ll}
0 & I_{n} \otimes\left[\begin{array}{lll}
0 & -1 & 0
\end{array}\right]
\end{array}\right] \text {. }
\end{aligned}
$$

We measure the performance of a decentralized affine controller according to the expected Euclidean distance between the desired and the induced vector of squared voltage magnitudes. In particular, we define the desired vector of voltage magnitudes as $v_{i}=v_{0}$ for all $i \in \mathcal{V}$.
Accordingly, we define the decentralized control design problem as:

$$
\left.\begin{array}{ll}
\operatorname{minimize} & \mathbf{E}\left[\left\|\boldsymbol{v}^{2}-v_{0}^{2} 1\right\|_{2}^{2}\right] \\
\text { subject to } & Q^{I} \in S, \\
& \boldsymbol{v}^{2}=R \boldsymbol{p}+X \boldsymbol{q}+v_{0}^{2} 1, \\
& \boldsymbol{p}=P \boldsymbol{\xi}, \\
& \boldsymbol{q}=Q \boldsymbol{\xi}+Q^{I} \boldsymbol{\xi}, \\
& \left|Q^{I} \xi\right| \leq \bar{s}^{I}-A \xi^{I} \\
& \underline{v}^{2} \leq v^{2} \leq \bar{v}^{2}
\end{array}\right\} \forall \xi \in \Xi,
$$

where the decision variables are the matrix $Q^{I}$ and the random vectors $\boldsymbol{v}^{2}, \boldsymbol{p}$, and $\boldsymbol{q}$. In what follows, we write problem (17) more concisely by eliminating the decision variables $\boldsymbol{v}^{2}, \boldsymbol{p}$, and $\boldsymbol{q}$. First note that $\boldsymbol{v}^{2}$ can be equivalently written as the following affine function of the system disturbance $\boldsymbol{\xi}$ :

$$
\boldsymbol{v}^{2}=V \boldsymbol{\xi}+v_{0}^{2} 1
$$

where the matrix $V \in \mathbf{R}^{n \times N_{\xi}}$ is given by

$$
V=R P+X Q+X Q^{I} \text {. }
$$

Additionally, we represent the $m=4 n$ robust linear constraints in problem (17) more succinctly as

$$
F Q^{I} \xi+G \xi \leq 0
$$

where the matrices $F$ and $G$ can be constructed from the underlying problem data. Their exact specification is given in Appendix A. We can now write problem (17) more compactly as

$$
\begin{array}{ll}
\operatorname{minimize} & \mathbf{E}\left[\|V \boldsymbol{\xi}\|_{2}^{2}\right] \\
\text { subject to } & Q^{I} \in S, V \in \mathbf{R}^{n \times N_{\xi}}, \\
& V=R P+X Q+X Q^{I}, \\
& F Q^{I} \xi+G \xi \leq 0 \quad \forall \xi \in \Xi .
\end{array}
$$

The decentralized control design problem (20) amounts to a robust convex program, which can be solved offline. The decentralized affine control policy computed according to problem (20) can then be implemented online in a fully decentralized fashion without requiring explicit communication between PV inverters. We refer the reader to Section IV for a detailed description of the online controller implementation.

\section{Conic Programming Reformulation}

The robust convex program (20) is seemingly intractable due to the infinite number of linear constraints that must be enforced. However, given our assumption that the support $\Xi$ of the system disturbance admits a conic representation, one can leverage on strong duality of conic linear programs 
to equivalently reformulate the robust convex program (20) as a finite-dimensional conic program-a classical technique from robust optimization. Specifically, one can prove the following result using [32, Thm. 3.2], and the fact that $\mathbf{E}\left[\|V \boldsymbol{\xi}\|_{2}^{2}\right]=\mathbf{E}\left[\operatorname{Tr}\left(V^{\top} V \boldsymbol{\xi} \boldsymbol{\xi}^{\top}\right)\right]=\operatorname{Tr}\left(V^{\top} V M\right)$.

Theorem 1. Let $Q^{I^{*}}$ be an optimal solution to the following finite-dimensional conic program:

$$
\begin{array}{ll}
\text { minimize } & \operatorname{Tr}\left(M V^{\top} V\right) \\
\text { subject to } & Q^{I} \in S, V \in \mathbf{R}^{n \times N_{\xi}}, \\
& \Pi \in \mathbf{R}^{\ell \times m}, \quad \nu \in \mathbf{R}_{+}^{m}, \\
& V=R P+X Q+X Q^{I}, \\
& F Q^{I}+G+\nu e_{1}^{\top}+\Pi^{\top} W=0, \\
& \Pi \succeq \mathcal{K}^{*} 0 .
\end{array}
$$

It follows that $Q^{I^{*}}$ is an optimal solution to problem (20).

Several comments are in order. First, the specification of the conic program (21) relies on the probability distribution of the disturbance $\xi$ only through its support $\Xi$ and second-order moment matrix $M$. Second, this conic program can be efficiently solved for a variety of cones $\mathcal{K}$, including polyhedral and second-order cones. In particular, problem (21) amounts to a quadratic program if $\mathcal{K}$ is a polyhedral cone, and a second-order cone program if $\mathcal{K}$ is a second-order cone.

\section{REAL-TIME CONTROLLER IMPLEMENTATION}

The implementation of the controller designed according to Theorem 1 relies on the assumption that the statistics of the system disturbance remain unchanged within the time interval during which the decentralized controller is deployed and implemented. This assumption might not hold in practice. In what follows, we present a method to the offline design and online implementation of the decentralized controller, when the statistics of the system disturbance vary at an (arbitrarily) fast time-scale. In the offline design, we compute the decentralized controller via the solution of a robust convex program, which takes into account the time variation in the statistics of the system disturbance. In the online implementation, the controller we design yields reactive power injection profiles that are guaranteed to robustly satisfy the network voltage and inverter capacity constraints at the fast time-scale.

\section{A. Real-time System Disturbance Model}

We begin by describing the real-time system disturbance as a discrete-time stochastic process over time periods indexed by $t=1,2, \ldots$ Each discrete time period is defined over a time interval of length $\Delta$. Accordingly, we denote by $\boldsymbol{\xi}(t)$ the system disturbance during time period $t$, which is modeled as a random vector with known second-moment and support. Similar to our development of the uncertainty model in Section II-D, we assume that the random vector $\boldsymbol{\xi}(t)$ has support $\Xi(t)$ that is convex, compact, and representable as

$$
\Xi(t)=\left\{\xi(t) \in \mathbf{R}^{N_{\xi}} \mid e_{1}^{\top} \xi(t)=1 \text { and } W(t) \xi(t) \succeq_{\mathcal{K}} 0\right\} \text {, }
$$

where the matrix $W(t) \in \mathbf{R}^{\ell \times N_{\xi}}$ is known. The secondorder moment matrix of the random vector $\boldsymbol{\xi}(t)$

$$
M(t)=\mathbf{E}\left[\boldsymbol{\xi}(t) \boldsymbol{\xi}(t)^{\top}\right]
$$

is assumed to be positive definite and finite-valued.

\section{B. Control Design to Enable Real-time Implementation}

The support and second-order moment matrix of the system disturbance might vary at a fast time-scale of seconds to minutes. In general, this corresponds to a time interval that is much shorter than the time interval during which a decentralized controller is deployed and implemented. In order to ensure its robust constraint satisfaction in real-time, the decentralized controller needs to be designed in anticipation of the non-stationarity in the statistics of the underlying system disturbance. In what follows, we formally present the problem of designing a static robust decentralized controller that is implemented over multiple discrete time periods. Its optimal solution can be calculated via the solution of a finite-dimensional conic program.

More specifically, consider the problem of designing a decentralized affine controller that is implemented over discrete time periods $t=1, \ldots, T$. Our objective is to minimize the sum of expected voltage regulation error over the $T$ time periods, while guaranteeing the robust satisfaction of network voltage and inverter capacity constraints. This amounts to the following robust convex program:

$$
\begin{array}{ll}
\text { minimize } & \mathbf{E}\left[\sum_{t=1}^{T}\|V \boldsymbol{\xi}(t)\|_{2}^{2}\right] \\
\text { subject to } & Q^{I} \in S, V \in \mathbf{R}^{n \times N_{\xi}} \\
& V=R P+X Q+X Q^{I} \\
& F Q^{I} \xi+G \xi \leq 0 \quad \forall \xi \in \bigcup_{t=1}^{T} \Xi(t) .
\end{array}
$$

A challenge in the solution of robust program (22) derives from the potential non-convexity in the uncertainty set $\bigcup_{t=1}^{T} \Xi(t)$. In Theorem 2, we show that problem (22) admits an equivalent reformulation as a finite-dimensional conic program. Its proof mirrors on that of Theorem 1, and is, therefore, omitted due to space constraints. 
Theorem 2. Let $Q^{I^{*}}$ be an optimal solution to the following finite-dimensional conic program:

$$
\begin{array}{ll}
\operatorname{minimize} & \operatorname{Tr}\left(\bar{M} V^{\top} V\right) \\
\text { subject to } & Q^{I} \in S, V \in \mathbf{R}^{n \times N_{\xi}} \\
& \Pi(t) \in \mathbf{R}^{\ell \times m}, \quad \nu(t) \in \mathbf{R}_{+}^{m} \\
& V=R P+X Q+X Q^{I} \\
& F Q^{I}+G+\nu(t) e_{1}^{\top}+\Pi(t)^{\top} W(t)=0 \\
& \Pi(t) \succeq \mathcal{K}^{*} 0 \\
& t=1, \ldots, T,
\end{array}
$$

where the matrix $\bar{M}$ is defined according to

$$
\bar{M}=\sum_{t=1}^{T} M(t) .
$$

It follows that $Q^{I^{*}}$ is an optimal solution to problem (22).

Remark 1 (Communication Requirements). We remark that the communication required for the implementation of the decentralized controller $Q^{I^{*}}$ is low. More precisely, assuming that the decentralized affine controller $Q^{I^{*}}$ is computed at a central computer, four real numbers need to be transmitted to each PV inverter. This communication requirement is clearly independent of the time horizon $T$.

A potential drawback of the optimization problem (23) is that the number of decision variables grows linearly with $T$, in general. Still, one can compute a feasible solution to problem (22) in time that is independent of the time horizon $T$ via the construction of a conservative inner approximation. We provide such a conservative inner approximation in the following corollary to Theorem 1.

Corollary 1. Consider the set $\widehat{\Xi} \subseteq \mathbf{R}^{N_{\xi}}$ given by

$$
\widehat{\Xi}=\left\{\xi \in \mathbf{R}^{N_{\xi}} \mid e_{1}^{\top} \xi=1 \text { and } \widehat{W} \xi \succeq_{\mathcal{K}} 0\right\},
$$

where the matrix $\widehat{W} \in \mathbf{R}^{k \times N_{\xi}}$ is known, and $\widehat{\Xi}$ satisfies

$$
\widehat{\Xi} \supseteq \bigcup_{t=1}^{T} \Xi(t) .
$$

Let $\bar{Q}^{I}$ be an optimal solution to the following finitedimensional conic program:

$$
\begin{array}{ll}
\text { minimize } & \operatorname{Tr}\left(\bar{M} V^{\top} V\right) \\
\text { subject to } & Q^{I} \in S, \Pi \in \mathbf{R}^{k \times m}, \quad \nu \in \mathbf{R}_{+}^{m} \\
& V=R P+X Q+X Q^{I} \\
& F Q^{I}+G+\nu e_{1}^{\top}+\Pi^{\top} \widehat{W}=0 \\
& \Pi \succeq \mathcal{K}^{*} 0 .
\end{array}
$$

It follows that $\bar{Q}^{I}$ is a feasible solution to problem (22).
In practice, it is always possible to construct the set $\widehat{\Xi}$ such that condition (24) is satisfied. For example, one can construct the set $\widehat{\Xi}$ based on the power rating of loads and the capacity constraints of PV inverters. In particular, such a construction of the set $\widehat{\Xi}$ yields a finite-dimensional conic program whose dimension is independent of the time horizon $T$.

We conclude this section with a discussion on the implementation of decentralized affine controllers computed according to Corollary 1. First, its implementation yields control inputs over the discrete time periods $t=1, \ldots, T$ that are guaranteed to be robustly feasible. Second, as the solution of problem (25) does not require a priori knowledge of the realization of system disturbance, it can be solved offline using only the statistics of the system disturbance. In particular, this implies that the decentralized controller does not have to be computed in real-time.

\section{Case Study}

We demonstrate the performance of our proposed decentralized affine controller on a three-phase balanced version of the IEEE 123-node test feeder [33]. Its single-line diagram is presented in Figure 2. We operate the system over a time horizon of 24 hours, beginning at 12 AM.

\section{A. System Decription}

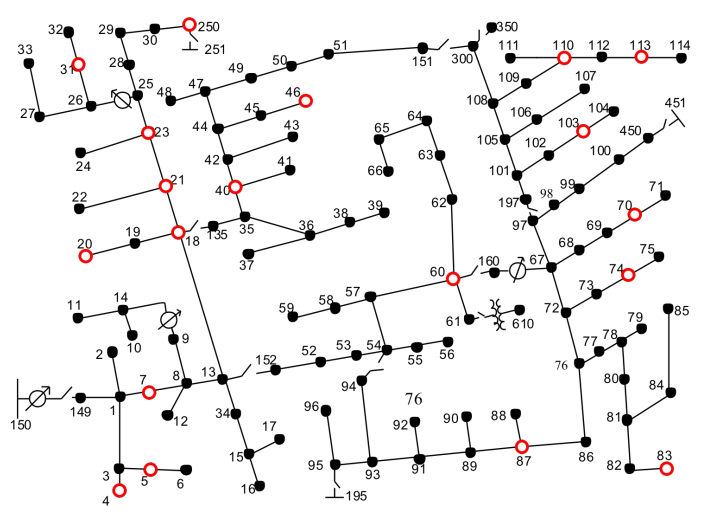

Fig. 2: Schematic diagram of the IEEE 123-node test feeder. Each red empty node has installed a PV inverter with active power capacity $0.8 \mathrm{MW}$.

A PV inverter that is capable of supplying up to $0.8 \mathrm{MW}$ of active power is installed at each of the red empty nodes in Figure 2. In Table I, we specify the parameter values of the network voltage magnitude and PV inverter capacity constraints. The tap positions of all voltage regulators are set to the center. That is, the turns ratio equals 1 for each voltage regulator. As for the random load processes, we plot the expected total system load trajectories in Figure 3. 


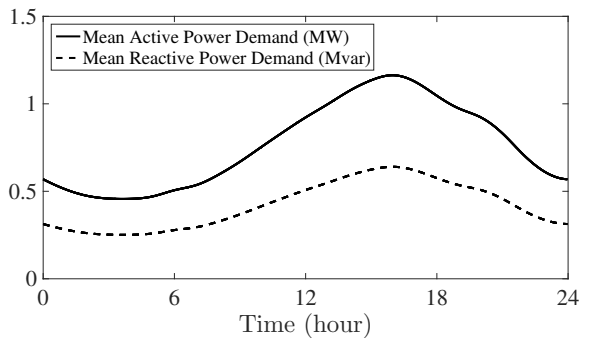

Fig. 3: The above figure depicts the expected total system load (active and reactive power) trajectories.

TABLE I: Specification of system data.

\begin{tabular}{ll}
\hline Feeder specifications & \\
\hline Base voltage magnitude & $4.16 \mathrm{kV}$ \\
Substation voltage magnitude & $v_{0}=1$ (per-unit) \\
Voltage magnitude constraints & $\underline{v}=0.95 \cdot 1, \bar{v}=1.05 \cdot 1$ (per-unit) \\
\hline PV inverter at node $i$ & \\
\hline Apparent power capacity & $\bar{s}_{i}^{I}=1$ (MVA) \\
Max active power supply & $\bar{p}_{i}^{I}(t)=\max \left\{0.8 \sin \left(\frac{\Delta \pi t}{720}-\frac{\pi}{2}\right), 0\right\}$ \\
Active power supply & $\boldsymbol{\xi}_{i}^{I}(t) \sim \operatorname{Uni}\left[0, \bar{p}_{i}^{I}(t)\right](\mathrm{MW})$ \\
\hline
\end{tabular}

The exact specification of the nodal demand processes is omitted due to space constraints. Additionally, we assume that for each discrete time period $t$, the system disturbance $\boldsymbol{\xi}(t)$ consists of $N_{\xi}$ random variables that are mutually uncorrelated.

We assume that the system disturbance stays constant in every 2-minute interval. Accordingly, each discrete time period corresponds to a time interval of length $\Delta=2$ minutes. The decentralized affine controllers we use are computed according to Corollary 1. The decentralized affine controller is updated every 10 minutes by the central computer.

\section{B. Numerical Results and Discussion}

We begin by demonstrating the effectiveness of the proposed decentralized affine controller in regulating voltage. We do so by comparing the nodal voltage trajectories in the distribution system with and without control. In Figure 4(a), we plot a particular realization and the empirical confidence interval of the trajectories of nodal voltage magnitudes that materialize under the decentralized controller computed according to Corollary 1. In Figure 4(b), we plot their uncontrolled counterparts. First, note that in the uncontrolled distribution system, the large amount of active power supply from PV inverters manifests in overvoltage in the distribution network. Additionally, the nodal voltage magnitudes in the uncontrolled distribution system exhibit large fluctuations as a consequence of the large fluctuation in the active power supplied from PV inverters. On the contrary, the nodal voltage magnitudes in the controlled distribution network are always feasible, and are regulated close to 1 per-unit throughout the entire day with high probability.

In Figure 5, we illustrate the behavior of the trajectories of PV reactive power injections generated by the decentralized controller. First, notice that the reactive power injections from PV inverters exhibit large fluctuations during the daytime hours. This is a consequence of the large fluctuations in the active power supplied from PV inverters. In particular, an increase in active power supplied from $\mathrm{PV}$ inverters increases the voltage magnitudes across the network. In order to suppress the rise and fall in voltage, the decentralized affine controller generates reactive power injections from PV inverters that are negatively correlated with their active power supply. More interestingly, there is a dip in the maximum reactive power consumption from PV inverters during the middle of the day. Note that the active power supply from PV inverters is at peak during the middle of the day. This serves to limit the reactive power capacity available to the PV inverter during those times. As a result, the reactive power capacity constraint (11) is binding during the middle of the day, which leads to the dip in the maximum reactive power being absorbed by the PV inverters.

\section{CONCLUSION}

In this paper, we consider the decentralized reactive power control of photovoltaic (PV) inverters to regulate distribution system voltage profile subject to voltage magnitude and PV inverter capacity constraints. Our approach involves the offline design and the online implementation of the decentralized controller. In the offline control design, we devise a method to compute a decentralized affine controller through the solution of a finite-dimensional conic program. In the online implementation, the resulting affine controller can be implemented over a fast timescale, and yields nodal voltage and PV reactive power injection trajectories that are guaranteed to be feasible for all realizations of the system disturbance. We demonstrate the ability of our control design technique to regulate voltage profile effectively with a study of the IEEE 123node test feeder.

There are several interesting directions for future work. For example, a practical drawback of our approach is its explicit reliance on the assumption of constant power loads. Such a load model does not capture the class of voltage-dependent loads, e.g., heating and lighting loads, induction motors, and shunt capacitors. In the future, it would be of interest to extend the formulation in this paper to accommodate a mixture of constant power and voltage-dependent loads. Additionally, all of our results 

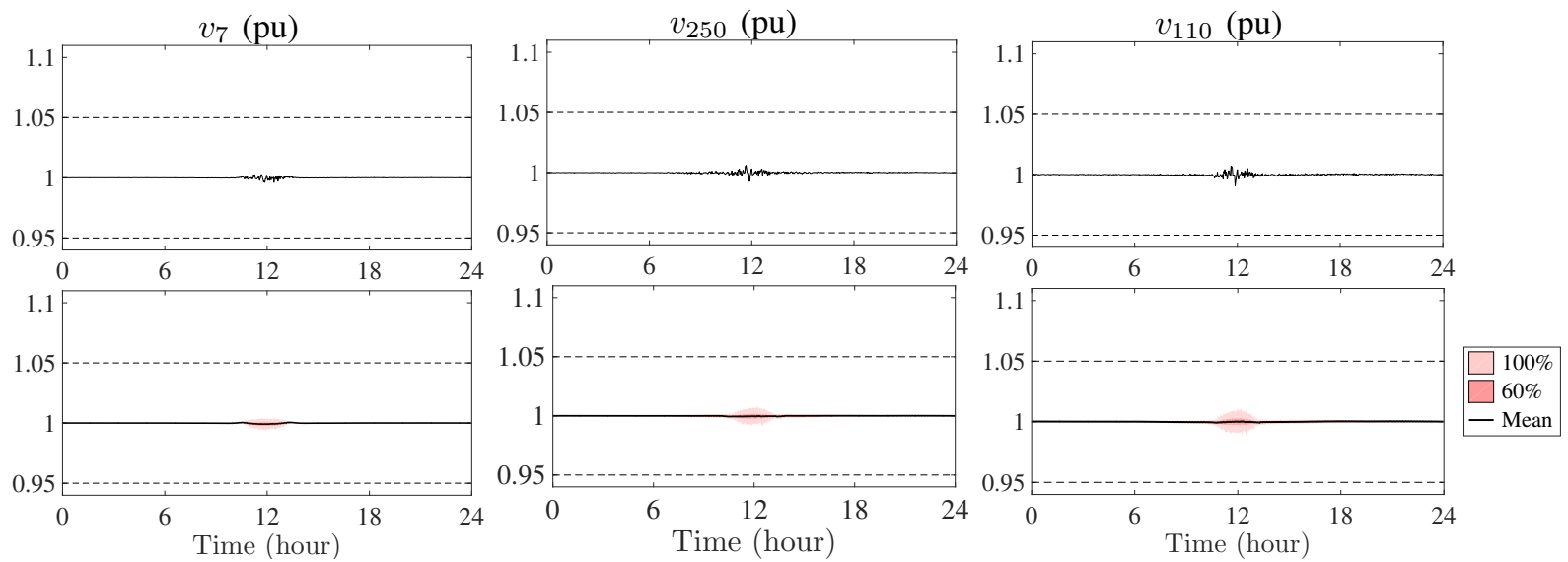

(a) Nodal voltage magnitudes in a controlled distribution system.
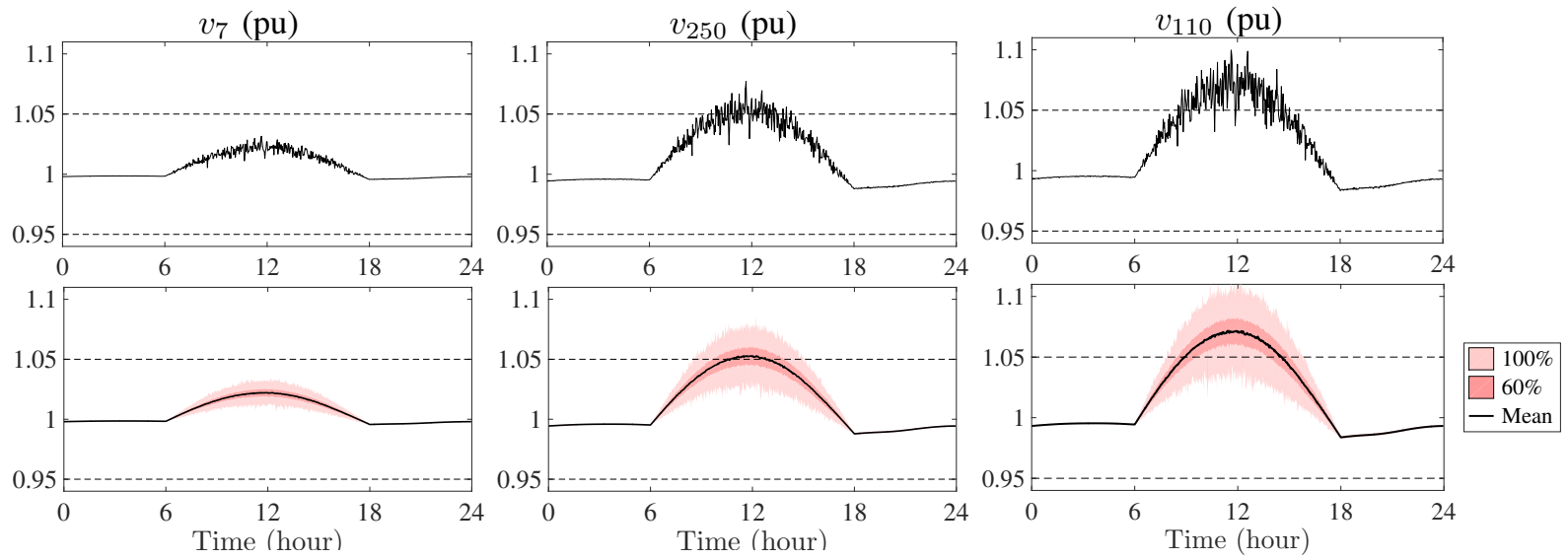

(b) Nodal voltage magnitudes in an uncontrolled distribution system.

Fig. 4: The figures in the first and third rows depict a particular realization of nodal voltage magnitude trajectories in (a) a controlled distribution system operated under the decentralized affine controller, and (b) an uncontrolled distribution system. The figures in the second and fourth rows depict the empirical confidence intervals associated with these trajectories. The empirical confidence intervals are estimated using 500 independent realizations of the system disturbance. The black dashed lines specify voltage magnitudes limits.
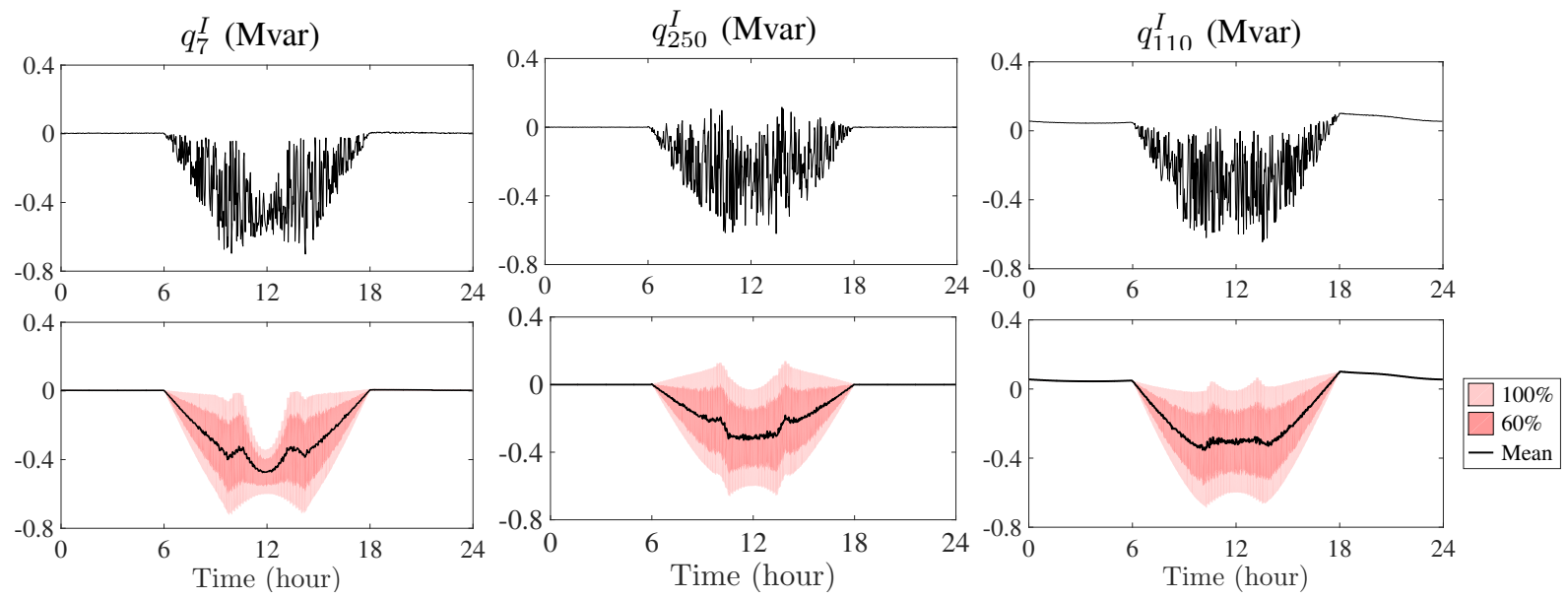

Fig. 5: The figures in the first row depict a particular realization of the PV reactive power injection trajectories at node 7,250 , and 110, respectively. The figures in the second row depict the empirical confidence intervals associated with these trajectories. They are estimated using 500 independent realizations of the system disturbance. 
rely on the assumption that the distribution system is threephase balanced. Such an assumption is unlikely to hold in practice. It would be of interest to extend our techniques to accommodate unbalanced distribution systems.

\section{REFERENCES}

[1] California ISO, "Daily renewables watch for $05 / 13 / 2017$, ," available at: http://content.caiso.com/green/renewrpt/20170513_ DailyRenewablesWatch.pdf.

[2] M. Thomson and D. Infield, "Impact of widespread photovoltaics generation on distribution systems," IET Renewable Power Generation, vol. 1, no. 1, pp. 33-40, 2007.

[3] V. Disfani, P. Ubiratan, and J. Kleissl, "Model predictive on-load tap changer control for high penetrations of PV using sky imager solar forecast," California Solar Initiative RD\&D Program, 2015.

[4] S. Corsi, Voltage control and protection in electrical power systems: from system components to wide-area control. Springer, 2015.

[5] M. Farivar, R. Neal, C. Clarke, and S. Low, "Optimal inverter VAR control in distribution systems with high PV penetration," in Power and Energy Society General Meeting, 2012 IEEE. IEEE, 2012, pp. 1-7.

[6] E. Dall'Anese, G. B. Giannakis, and B. F. Wollenberg, "Optimization of unbalanced power distribution networks via semidefinite relaxation," in North American Power Symposium (NAPS), 2012. IEEE, 2012, pp. 1-6.

[7] Z. Shen and M. E. Baran, "Gradient based centralized optimal Volt/Var control strategy for smart distribution system," in Innovative Smart Grid Technologies (ISGT), 2013 IEEE PES. IEEE, 2013, pp. 1-6.

[8] E. Dall'Anese, S. V. Dhople, and G. B. Giannakis, "Optimal dispatch of photovoltaic inverters in residential distribution systems," IEEE Transactions on Sustainable Energy, vol. 5, no. 2, pp. 487497, 2014.

[9] K. Turitsyn, S. Backhaus, M. Chertkov et al., "Options for control of reactive power by distributed photovoltaic generators," Proceedings of the IEEE, vol. 99, no. 6, pp. 1063-1073, 2011.

[10] V. Kekatos, L. Zhang, G. B. Giannakis, and R. Baldick, "Voltage regulation algorithms for multiphase power distribution grids," IEEE Transactions on Power Systems, vol. 31, no. 5, pp. 39133923, 2016.

[11] N. Li, G. Qu, and M. Dahleh, "Real-time decentralized voltage control in distribution networks," in Communication, Control, and Computing (Allerton), 2014 52nd Annual Allerton Conference on. IEEE, 2014, pp. 582-588.

[12] P. D. Ferreira, P. Carvalho, L. A. Ferreira, and M. D. Ilic, "Distributed energy resources integration challenges in low-voltage networks: Voltage control limitations and risk of cascading," Sustainable Energy, IEEE Transactions on, vol. 4, no. 1, pp. 82-88, 2013.

[13] H. Zhu and H. J. Liu, "Fast local voltage control under limited reactive power: Optimality and stability analysis," IEEE Transactions on Power Systems, vol. 31, no. 5, pp. 3794-3803, 2016.

[14] P. Jahangiri and D. C. Aliprantis, "Distributed Volt/Var control by PV inverters," IEEE Transactions on power systems, vol. 28, no. 3, pp. 3429-3439, 2013.

[15] H.-G. Yeh, D. F. Gayme, and S. H. Low, "Adaptive VAR control for distribution circuits with photovoltaic generators," IEEE Transactions on Power Systems, vol. 27, no. 3, pp. 1656-1663, 2012.

[16] M. Farivar, L. Chen, and S. Low, "Equilibrium and dynamics of local voltage control in distribution systems," in Decision and Control (CDC), 2013 IEEE 52nd Annual Conference on. IEEE, 2013, pp. 4329-4334.

[17] B. Zhang, A. Lam, A. D. Domínguez-García, and D. Tse, "An optimal and distributed method for voltage regulation in power distribution systems," Power Systems, IEEE Transactions on, vol. 30, no. 4, pp. 1714-1726, 2015.

[18] H. J. Liu, W. Shi, and H. Zhu, "Distributed voltage control in distribution networks: Online and robust implementations," IEEE Transactions on Smart Grid, 2017.
[19] S. S. Guggilam, E. DallAnese, Y. C. Chen, S. V. Dhople, and G. B. Giannakis, "Scalable optimization methods for distribution networks with high PV integration," IEEE Transactions on Smart Grid, vol. 7, no. 4, pp. 2061-2070, 2016.

[20] D. B. Arnold, M. Negrete-Pincetic, M. D. Sankur, D. M. Auslander, and D. S. Callaway, "Model-free optimal control of VAR resources in distribution systems: an extremum seeking approach," IEEE Transactions on Power Systems, vol. 31, no. 5, pp. 3583-3593, 2016.

[21] B. Robbins, C. N. Hadjicostis, A. D. Domínguez-García et al., "A two-stage distributed architecture for voltage control in power distribution systems," Power Systems, IEEE Transactions on, vol. 28, no. 2, pp. 1470-1482, 2013.

[22] S. Bolognani and S. Zampieri, "A distributed control strategy for reactive power compensation in smart microgrids," Automatic Control, IEEE Transactions on, vol. 58, no. 11, pp. 2818-2833, 2013.

[23] S. Bolognani, R. Carli, G. Cavraro, and S. Zampieri, "Distributed reactive power feedback control for voltage regulation and loss minimization," IEEE Transactions on Automatic Control, vol. 60, no. 4, pp. 966-981, 2015.

[24] V. Kekatos, G. Wang, A. J. Conejo, and G. B. Giannakis, "Stochastic reactive power management in microgrids with renewables," Power Systems, IEEE Transactions on, vol. 30, no. 6, pp. 3386$3395,2015$.

[25] R. A. Jabr, "Linear decision rules for control of reactive power by distributed photovoltaic generators," IEEE Transactions on Power Systems, 2017.

[26] M. E. Baran and F. F. Wu, "Optimal capacitor placement on radial distribution systems," Power Delivery, IEEE Transactions on, vol. 4, no. 1 , pp. 725-734, 1989.

[27] — "Optimal sizing of capacitors placed on a radial distribution system," Power Delivery, IEEE Transactions on, vol. 4, no. 1, pp. 735-743, 1989.

[28] — - "Network reconfiguration in distribution systems for loss reduction and load balancing," Power Delivery, IEEE Transactions on, vol. 4, no. 2, pp. 1401-1407, 1989.

[29] International Electrotechnical Commission et al., Efficient Electrical Energy Transmission and Distribution. IEC, 2007.

[30] W. Lin and E. Bitar, "Performance bounds for robust decentralized control," in 2016 American Control Conference (ACC). IEEE, 2016, pp. 4323-4330.

[31] Y.-C. Ho, "Team decision theory and information structures," Proceedings of the IEEE, vol. 68, no. 6, pp. 644-654, 1980.

[32] A. Ben-Tal, A. Goryashko, E. Guslitzer, and A. Nemirovski, "Adjustable robust solutions of uncertain linear programs," Mathematical Programming, vol. 99, no. 2, pp. 351-376, 2004.

[33] Distribution Test Feeder Working Group, "Distribution test feeders," available at: https://ewh.ieee.org/soc/pes/dsacom/testfeeders/.

\section{APPENDIX A \\ MATRIX DEFINITIONS}

The matrices $F$ and $G$ used in Eq. (19) are specified according to

$F=\left[\begin{array}{c}X \\ -X \\ I_{n} \\ -I_{n}\end{array}\right]$,

$G=\left[\begin{array}{cc}v_{0}^{2} 1-\bar{v}^{2} & 0 \\ \underline{v}^{2}-v_{0}^{2} 1 & 0 \\ -\bar{s}^{I} & A \otimes\left[\begin{array}{lll}0 & 0 & 1\end{array}\right] \\ -\bar{s}^{I} & A \otimes\left[\begin{array}{lll}0 & 0 & 1\end{array}\right]\end{array}\right]+\left[\begin{array}{c}R P+X Q \\ -(R P+X Q) \\ 0 \\ 0\end{array}\right]$. 